\title{
Coupling gauge theory to spinfoam 3d quantum gravity
}

\author{
Simone Speziale* \\ Perimeter Institute, 31 Caroline St. N, Waterloo, ON N2L 2Y5, Canada.
}

June 11, 2007

\begin{abstract}
We construct a spinfoam model for Yang-Mills theory coupled to quantum gravity in three dimensional riemannian spacetime. We define the partition function of the coupled system as a power series in $g_{0}^{2} G$ that can be evaluated order by order using grasping rules and the recoupling theory. With respect to previous attempts in the literature, this model assigns the dynamical variables of gravity and Yang-Mills theory to the same simplices of the spinfoam, and it thus provides transition amplitudes for the spin network states of the canonical theory. For SU(2) Yang-Mills theory we show explicitly that the partition function has a semiclassical limit given by the Regge discretization of the classical Yang-Mills action.
\end{abstract}

\section{Contents}

1 Introduction $\quad 1$

2 The classical theory 2

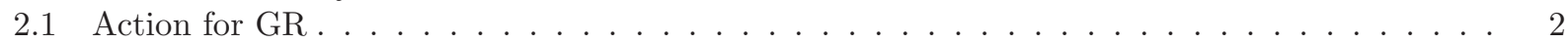

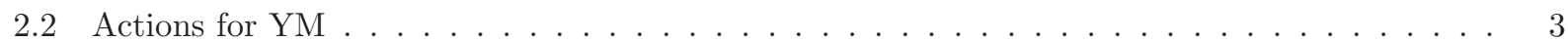

3 Discretisation $\quad 4$

4 Spinfoams and quantisation of BF theory 6

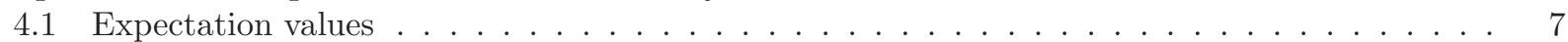

5 The semiclassical limit $\quad 9$

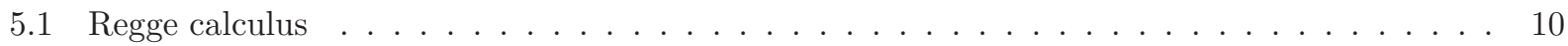

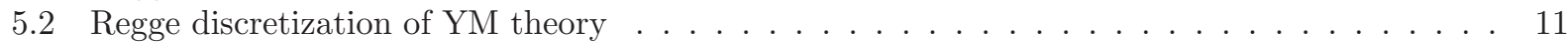

6 Spinfoam model of the coupled system 12

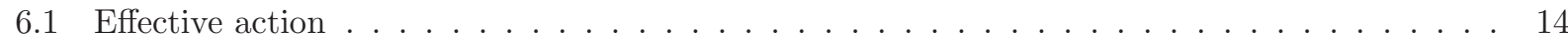

6.2 Tetrahedral asymptotics of the coupled partition function $\ldots \ldots \ldots \ldots \ldots$

7 Conclusions $\quad 16$

\section{Introduction}

The large spin limit has proven to be a useful tool to study the semiclassical physics of the spinfoam formalism for loop quantum gravity [1]. Recent developments include the right spacetime dependence of the free graviton propagator [2]. On a fixed spinfoam, this limit is related to the emergence of Regge calculus [3], a discrete approximation to General Relativity (GR). This result is very desirable, as all physically interesting quantum field theories have useful discrete approximations [4]. In particular, this limit is transparent in the Ponzano-Regge (PR) model for three dimensional riemannian quantum gravity [5]: the weights of the spinfoam partition function are, in the large spin limit, exponentials of the Regge action. Similar results

*sspeziale@perimeterinstitute.ca 
are expected in four dimensions [6,7]. If this is the correct way of studying semiclassical physics, then it should be applied not only to the partition function, but also to expectation values and to the coupling with matter. In [8] it was indeed shown that expectation values of geometric quantities in the PR model tend exactly to their classical values in this limit. In this paper we continue along this line of investigation, and we construct a coupled spinfoam model of GR and Yang-Mills (YM) theory in 3d riemannian spacetime that we show has the right semiclassical limit.

The relevance of the result concerns also the consistence of the proposed coupling of matter fields to spinfoam quantum gravity. In fact, the literature on the argument is somewhat limited. ${ }^{1}$ Models have been constructed specifically for YM fields [13], but they suffer from the following problem: the discretization procedure at the root of the quantization treats the gravitational and YM connections in a different way. The advantage of doing so is that the geometric information of the quantum gravity sector becomes directly available to define the metric dependence of the YM action to be quantized. The drawback is that it leads to a spinfoam model where the dynamical variables for GR and YM are attached inhomogeneously to the triangulation discretizing the spacetime manifold, thus the model does not provide transition amplitudes for the canonical theory: the data carried by the spin network states of the canonical theory would not match the data carried by a $2 \mathrm{~d}$ slice of the spinfoam.

In this paper we show how one can consistently couple YM theory discretizing both the gravitational and YM holonomies in the same way. The key to do so is the use of the generating functional techniques introduced in [14]. The same procedure has been recently applied to couple fermionic fields to PR [15]. As a consequence of this procedure, the metric dependence of the YM sector is given by a quantum operator whose action can be evaluated using the recoupling theory for $\mathrm{SU}(2)$. We study in detail this operator and show that the model has the right semiclassical limit but also non trivial quantum corrections. The resulting model describes spinfoams with labels attached homogeneously, thus it provides transition amplitudes for the canonical theory.

\section{The classical theory}

\subsection{Action for GR}

We represent spacetime by a $3 \mathrm{~d}$ differentiable manifold $M$, and we use the triad field as fundamental variable for GR, as this is more suitable to the spinfoam formalism. The triad is an $\mathrm{SU}(2)$-valued 1-form $e_{\mu}^{I}(x)$ related to the metric by

$$
g_{\mu \nu}(x)=e_{\mu}^{I}(x) e_{\nu}^{J}(x) \delta_{I J} .
$$

Geometrically, the triad is a homomorphism between the tangent bundle $T(M)$ and a principal bundle $P(M, \mathrm{SU}(2))$ whose fibres take values in the $\mathrm{SU}(2)$ group. $^{2}$ Over the bundle $P(M, \mathrm{SU}(2))$ we define a connection 1-form $\omega_{\mu}^{I J}(x)$, with curvature $F(\omega)$.

We use the following first order action for GR,

$$
S_{\mathrm{GR}}\left[e_{\mu}^{I}(x), \omega_{\mu}^{I J}(x)\right]=\frac{1}{8 \pi G} \int_{M} \operatorname{Tr} e \wedge F(\omega) .
$$

The trace $\operatorname{Tr}$ is over the algebra indices. The variation with respect to the connection gives the torsion-free condition, $d_{\omega} e=0$, as an equation of motion. Therefore $\omega$ is the spin connection. The variation with respect to the triad gives $F(\omega)=0$. The theory is invariant under diffeomorphisms acting on the (greek letters) spacetime indices, and $\mathrm{SU}(2)$ transformations acting on the (latin letters) algebra indices. Under these transformations, all solutions of the equations of motion are equivalent, thus the theory has no local degrees of freedom [17]. Indeed 3d GR is the topological BF theory with SU(2) as structure group [18].

We work with dimensionless coordinates, so that $\omega$ and $F$ are dimensionless and the triad field has dimensions of a length. We use units $c=1$, but we keep both $\hbar$ and $G$. The physical dimensions of the

\footnotetext{
${ }^{1}$ On the other hand, there are very promising results on the coupling of particles [9, 10]. See [11] for the coupling of matter in the canonical formalism, and [12] in the group field theory approach.

${ }^{2}$ In principle, the triad could take values in $S O(3)$, which is the proper structure group of pure $3 \mathrm{~d}$ riemannian GR. The group is extended to $\mathrm{SU}(2) \simeq \mathbb{Z}_{2} \times S O(3)$, in order to allow the coupling with fermions. Note also that while the bundle $P(M, \mathrm{SU}(2))$ is necessarily trivial, there are two isomorphism classes of the bundle $P(M, S O(3))$, characterized by their Stiefel-Whitney number, only one of which is isomorphic to the tangent bundle of $M$. For a discussion of a possible role of the topology of the bundle, as well as the constants $\hbar$ and $G$, see [16].
} 
gravitational constant $G$ depend on the spacetime dimension $n$. In SI units, we have $[G]=\mathrm{kg}^{-1} \mathrm{~m}^{n-3}$. In $3 \mathrm{~d}[G]=\mathrm{kg}^{-1}$ and thus (2) has the correct physical dimensions of an action, namely $\mathrm{kg} \cdot \mathrm{m}$, in units $c=1$. We define the $3 \mathrm{~d}$ Planck length as $\ell_{\mathrm{P}}=8 \pi \hbar G$.

\subsection{Actions for $\mathrm{YM}$}

Consider YM theory with gauge group $\mathcal{G}=\mathrm{SU}(N)$. We call $A_{\mu}^{a}$ the YM connection. The index $a$ is in the algebra $\mathfrak{g}$ of $\mathcal{G}$. The YM curvature is $F_{\mu \nu}^{a}=\partial_{\mu} A_{\nu}^{a}-\partial_{\nu} A_{\mu}^{a}+f^{a b c} A_{\mu}^{b} A_{\nu}^{c}$, where $f^{a b c}$ are the gauge group structure constants. Using the metric as the variable for the gravitational field, the Yang-Mills action on $n$-dimensional curved space is

$$
S_{\mathrm{YM}}\left[g_{\mu \nu}, A_{\mu}^{a}\right]=\frac{1}{4 g_{0}{ }^{2}} \int d^{n} x \sqrt{g} g^{\mu \rho} g^{\nu \sigma} F_{\mu \nu}^{a} F_{\rho \sigma}^{a} .
$$

Here $g \equiv \operatorname{det} g_{\mu \nu}$. The YM connection is dimensionless, thus the coupling constant $g_{0}$ has dimensions of an inverse action times $(n-4) / 2$ length dimensions (in order for the action $(3)$ to have the correct physical dimensions for every spacetime dimension $n$ ). In the $3 \mathrm{~d}$ case, we have $\left[\hbar^{-1} g_{0}{ }^{-2}\right]=[\hbar G]$. Consequentely we introduce a constant with dimensions of a length, called "YM length", $\ell_{\mathrm{YM}} \equiv \hbar^{-1} g_{0}{ }^{-2}$. The dimensionality of the YM coupling constant in 3d is well known, and it is for instance an indication of the super-renormalizability of this theory. As it will become clear below, the only relevant constant of the coupled system GR plus YM is the adimensional ratio $\ell_{\mathrm{P}} / \ell_{\mathrm{YM}}$.

To describe the coupled system with a unique set of variables, we recast this action in terms of the triad field (1). This can be done using twice the relation

$$
e \equiv \sqrt{g}=\operatorname{det} e_{\mu}^{I}(x)=\frac{1}{3 !} \varepsilon_{I J K} \varepsilon^{\mu \nu \rho} e_{\mu}^{I}(x) e_{\nu}^{J}(x) e_{\rho}^{K}(x)
$$

to write $(3)$ as

$$
S_{\mathrm{YM}}\left[e_{\mu}^{I}(x), A_{\mu}^{a}(x)\right]=\frac{1}{8 g_{0}^{2}} \int_{M} d^{3} x e^{-1} \varepsilon^{\mu \nu \rho} \varepsilon^{\lambda \sigma \tau} e_{\mu}^{I} e_{\lambda}^{I} F_{\nu \rho}^{a} F_{\sigma \tau}^{a} .
$$

Notice that no inverse triad $e_{I}^{\mu}$ appears in the action (5), at the price of having to deal with the inverse determinant $e^{-1}$.

The definition $T_{\mu}^{I}=\delta S_{\mathrm{YM}}[e, A] / \delta e_{I}^{\mu}$ gives the equations of motion,

$$
F_{\mu}^{I}(\omega)-\frac{1}{2} e_{\mu}^{I} F(\omega)=16 \pi G T_{\mu}^{I}, \quad \partial_{\mu} e_{\nu}^{I}-\partial_{\nu} e_{\mu}^{I}+\omega^{I}{ }_{J \mu} e_{\nu}^{J}-\omega_{J \nu}^{I} e_{\mu}^{J}=0, \quad D_{\mu}(A)\left(e F^{\mu \nu}(A)\right)=0 .
$$

As it is well known, the presence of matter does not add degrees of freedom to GR [17]. Namely the equations of motion can be solved without local degrees of freedom in the gravitational sector. Local degrees of freedom only come from the Yang-Mills sector of the theory. Geometrically, we are working with a bundle $P(M, \mathrm{SU}(2), \mathcal{G})$, where the fibres are independent. This follows from the fact that the YM field $A_{\mu}^{a}$ does not couple to the connection $\omega_{\mu}^{I J}$.

The coupled system of gravity and YM theory can be described using the action $S[e, \omega, A]$ given by (2) plus (5), where (2) describes gravity alone, while (5) describes both the YM field and the interaction with gravity. To achieve a more symmetric description, we consider an alternative formulation of YM theory, which makes use of a first order action: YM theory can be written as a deformation of BF theory [19]

$$
\frac{1}{2} \int d^{3} x \varepsilon^{\mu \nu \rho} B_{\mu}^{a} F_{\nu \rho}^{a}(A)-\frac{g_{0}^{2}}{2} \int d^{3} x \sqrt{g} g^{\mu \nu} B_{\mu}^{a} B_{\nu}^{a} .
$$

The auxiliary $B$ field has dimensions of an action. The classical equivalence with (3) can be easily checked by looking at the following equation of motion,

$$
0=\frac{\delta S}{\delta B_{\mu}^{a}}=\frac{1}{2} \varepsilon^{\mu \nu \rho} F_{\nu \rho}^{a}-g_{0}^{2} \sqrt{g} B_{a}^{\mu},
$$

and using $\varepsilon^{\mu \rho \sigma} \varepsilon^{\nu \lambda \tau} g_{\mu \nu}=g g^{\rho[\lambda} g^{\sigma \tau]}$.

At the quantum level, the formal equivalence can be straightforwardly verified performing the gaussian integration over the $B$ field in the partition function for (6). On the other hand, the equivalence of the 
perturbative quantization of (6) and (5) is rather subtle: particular care has to be taken in the gauge fixing procedure [20, 21]. These are relevant issues that will however not concern us in the following, where we focus only on defining the full spinfoam partition function and studying its semiclassical limit.

Introducing the Hodge star defined by $* B=\frac{1}{2 \sqrt{g}} \varepsilon^{\mu}{ }_{\nu \rho} B_{\mu} d x^{\nu} d x^{\rho}$ the interacting terms can be written as $B \wedge * B$, and the coupled action

$$
S\left[e_{\mu}^{I}(x), \omega_{\mu}^{I J}(x), B_{\mu}^{a}(x), A_{\mu}^{a}(x)\right]=\frac{1}{8 \pi G} \int_{M} \operatorname{Tr} e \wedge F(\omega)+\int_{M} \operatorname{Tr} B \wedge F(A)-\frac{g_{0}^{2}}{2} \int_{M} \operatorname{Tr} B \wedge * B .
$$

With abuse of notation, Tr denotes the trace over both algebraic indices $I$ and $a$. As for (5), the metricdependent interacting term can be written in terms of triads only using (4) twice,

$$
B \wedge * B=\sqrt{g} g^{\mu \nu} B_{\mu}^{a} B_{\nu}^{a}=\frac{1}{2} e^{-1} \varepsilon^{\mu \rho \sigma} \varepsilon^{\nu \lambda \tau} e_{\lambda}^{I} e_{\tau}^{J} e_{\rho}^{I} e_{\sigma}^{J} B_{\mu}^{a} B_{\nu}^{a}
$$

This is the action which we use in this paper to study quantum gravity coupled to YM theory: it has two free pieces, which correspond to topological theories, and an interacting piece with local degrees of freedom.

\section{Discretisation}

In the following, we are going to quantise the theory using functional integrals regularized by means of a discretisation of the spacetime manifold. ${ }^{3}$ To do so, we introduce an oriented triangulation $\Delta$. This is an abstract cellular complex made out of points $p$, segments $s$, triangles $t$ and tetrahedra $\tau$, together with an operation that allows the identification of the $n-1$ dimensional boundary of an $n$ dimensional object in $\Delta$. To this abstract complex is associated its dual $\Delta^{*}$, with a one-to-one correspondence between $n$-dimensional objects of $\Delta$ and $3-n$ dimensional objects of $\Delta^{*}$. The 2-skeleton $\mathcal{J}$ of $\Delta^{*}$ is a 2-complex made of called vertices $v$, edges $e$ and faces $f$.

The coupled action (7) is invariant under diffeomorphisms of $M$. Introducing a fixed triangulation breaks this symmetry, thus a procedure to restore it will be necessary in the final theory. A possible way of doing so is to use the group field theory formalism [23]. This formalism restores the original diffeomorphism invariance by producing in general a sum over triangulations weighted by the corresponding spinfoam amplitude for each fixed triangulation. We postpone the constuction of such a model for further work, and we focus here on the preliminary step of defining the model on a fixed triangulation.

On the abstract cellular complex $\Delta$, we introduce the algebra variables

$$
X_{s}^{I} \in \mathfrak{s} u(2), \quad B_{s}^{a} \in \mathfrak{g},
$$

associated to the segments, and the group variables

$$
g_{t} \in \mathrm{SU}(2), \quad U_{t} \in \mathcal{G},
$$

associated to the triangles. Furthermore, we define the following quantities:

$$
e^{Z_{s}} \equiv \mathcal{P} \prod_{t \ni s} g_{t}, \quad e^{W_{s}} \equiv \mathcal{P} \prod_{t \ni s} U_{t}
$$

Here $\mathcal{P}$ means that the product over all the triangles which share the same segment $s$ is oriented. The orientation is induced from the orientation of $s$.

Consider the classical (discrete) theory defined by the action

$$
S\left[X_{s}^{I}, g_{t}, B_{s}^{a}, U_{t}\right]=\hbar \sum_{s} \operatorname{Tr}\left[X_{s} Z_{s}\right]+\hbar \sum_{s} \operatorname{Tr}\left[B_{s} W_{s}\right]-\hbar \lambda S_{\mathrm{BB}}\left[X_{s}^{I}, B_{s}^{a}\right] .
$$

The interaction term is given by

$$
\hbar \lambda S_{\mathrm{BB}}\left[X_{s}^{I}, B_{s}^{a}\right]=\frac{1}{3} \pi \hbar^{3} g_{0}^{2} G \sum_{\tau} \frac{\mathcal{C}_{\tau}[X, B]}{\mathcal{V}_{\tau}[X]}
$$

\footnotetext{
${ }^{3}$ Notice that a lattice discretization of the action (6) on flat spacetime has been considered in [22].
} 
where we introduced the shorthand notations

$$
\mathcal{C}_{\tau}[X, B] \equiv \frac{1}{4} \sum_{p \in \tau} \sum_{s_{i}, t_{i} \in p} \varepsilon^{s_{1} s_{2} s_{3}} \varepsilon^{t_{1} t_{2} t_{3}} X_{s_{1}}^{I} X_{t_{1}}^{I} X_{s_{2}}^{J} X_{t_{2}}^{J} B_{s_{3}}^{a} B_{t_{3}}^{a},
$$

(here $\varepsilon^{s_{1} s_{2} s_{3}}$ is the completely antisymmetric tensor for the three segments in a given $p$, with the convention $\varepsilon^{123}=1$ for a right-handed triple) and

$$
\mathcal{V}_{\tau}[X] \equiv \frac{1}{4} \sum_{p \in \tau} \frac{1}{3 !} \varepsilon_{I J K} X_{1}^{I} X_{2}^{J} X_{3}^{K},
$$

where 123 is a right-handed triple of segments for each point $p$.

We kept the constants out of the definition of $S_{\mathrm{BB}}$ for a better clarity in later computations. The coupling constant between gravity and YM is

$$
\lambda=\frac{1}{3} \pi \hbar^{2} g_{0}^{2} G=\frac{1}{24} \frac{\ell_{\mathrm{P}}}{\ell_{\mathrm{YM}}} .
$$

The action (10) defines a theory on $\Delta$, with variables $X_{s}^{I}, g_{t}, B_{s}^{a}, U_{t}$. In the rest of this section, we will show that it is an approximation to the continuum theory with action (7). Recall that the latter is defined on $M$, with variables $e_{\mu}^{I}(x), \omega_{\mu}^{I J}(x), B_{\mu}^{a}(x), A_{\mu}^{a}(x)$. Consider an embedding $\iota: \Delta \rightarrow M$, which allows us to think of $\Delta$ as a cellular decomposition of $M$. Using the embedding, we have $\ell_{s}^{\mu} \sim \int_{s} d x^{\mu}$. For each point $p$ in each tetrahedron $\tau$, we call $\ell_{s}^{\mu}(p)$ the three vectors tangent to the three segments $s$ belonging to $p(\tau)$. We can think of $\ell_{s}^{\mu}(p)$ as a 3 by 3 matrix, and we choose coordinates such that $\operatorname{det} \ell_{s}^{\mu}(p)=\frac{1}{3 !} \varepsilon^{s_{1} s_{2} s_{3}} \varepsilon_{\mu \nu \rho} \ell_{s_{1}}^{\mu} \ell_{s_{2}}^{\nu} \ell_{s_{3}}^{\rho}=1$. The embedding $\iota$ pushes forward to an embedding for the dual triangulation $\Delta^{*}$, and we analogously have $\ell_{e}^{\mu} \sim \int_{e} d x^{\mu}$ for each edge in $\Delta^{*}$.

We make the following four identifications.

1. The triad field:

$$
e_{\mu}^{I}(x) \mapsto X_{s}^{I} \equiv \frac{1}{\ell_{\mathrm{P}}} e_{\mu}^{I}(x) \ell_{s}^{\mu} \sim \frac{1}{\ell_{\mathrm{P}}} \int_{s} e_{\mu}^{I}(x) d x^{\mu} .
$$

For each segment $s$ in $\Delta$, we clearly have $\ell_{s}^{2}=g_{\mu \nu} \ell_{s}^{\mu} \ell_{s}^{\nu}=\ell_{\mathrm{P}}^{2} X_{s}^{I} X_{s}^{I}$. Therefore the variables $X_{s}$ represent the segment lengths. We will see below that in the quantum theory they are observables with a discrete spectrum. This is the sense in which spinfoams describe a quantum geometry, with discrete properties for the geometrical observables.

2. The connection field:

$$
\omega_{\mu}^{I J}(x) \mapsto g_{t} \equiv e^{\omega_{\mu}^{I J} \ell_{e}^{\mu}} \sim e^{\int_{e} \omega^{I J}} .
$$

On the continuum, the connection is geometrically interpreted as an infinitesimal parallel transport. On a discrete setting, this should be properly taken over by a discrete minimal parallel transport. The group element defined above realizes this property.

3. The field $B$ :

$$
B_{\mu}^{a}(x) \mapsto B_{s}^{a} \equiv \frac{1}{\hbar} B_{\mu}^{a}(x) \ell_{s}^{\mu} \sim \frac{1}{\hbar} \int_{s} B_{\mu}^{a}(x) d x^{\mu} .
$$

The geometric interpretation of this auxiliary field is to provide lengths in the fibre space.

4. The YM connection:

$$
A_{\mu}^{a}(x) \mapsto U_{t} \equiv e^{A_{\mu}^{a} \ell_{e}^{\mu}} \sim e^{\int_{e} A^{a}} .
$$

As for the gravitational connection, the YM connection is discretised as a group element realizing a finite, though minimal, parallel transport along the edges of the dual triangulation.

We claim that for each configuration of the continuous fields we can find an embedding $\iota$ such that the difference between (7) and (10) is arbitrarily small, if the variables in (10) are interpreted as (15)-(18).

To see it, we start applying the Stokes' theorem to the connection around a closed face $f$ (for non-abelian groups, the theorem holds up to corrections in the area of the face):

$$
\sum_{e \in \partial f} \omega_{\mu} \ell_{e}^{\mu} \sim \int_{\partial f} \omega \approx \int_{f} F(\omega) .
$$


As a consequence of the equality above, the definitions (16) and (18) endow the group variables defined in (9) with the interpretation of holonomies,

$$
e^{Z_{s}} \sim e^{\int_{f} F(\omega)}, \quad e^{W_{s}} \sim e^{\int_{f} F(A)} .
$$

These group elements appear in the first two pieces of (10). When the embedding is sufficently refined, and the coordinate areas consequently small, we can expand the group elements around the algebra, so that $Z_{s} \simeq \int_{f} F(\omega)$ and the first two pieces of (10) reduce to the two BF terms of (7).

Consider now the interacting term (11). Let us choose a point $p$ in a tetrahedron, with 123 a right-handed triple of segments. Using (15) and $\operatorname{det} \ell_{s}^{\mu}(p)=1$, we immediately have

$$
\frac{1}{3 !} \varepsilon_{I J K} X_{1}^{I} X_{2}^{J} X_{3}^{K} \sim \frac{1}{\ell_{\mathrm{P}}^{3}} \frac{e}{3 !}=\frac{1}{\ell_{\mathrm{P}}^{3}} V_{\tau},
$$

where in the last step we used the fact that $e$ is the volume of the parallelepiped, and there are 3 ! tetrahedra in a parallelepiped. Symmetrizing the expression above over the four points of $\tau$ we get (13). Analogously, using (15), (17) and $\operatorname{det} \ell_{s}^{\mu}(p)=1$ we have

$$
\sum_{s_{i} \in p(\tau)} \varepsilon^{s_{1} s_{2} s_{3}} X_{s_{1}}^{I} X_{s_{2}}^{J} B_{s_{3}}^{a} \sim \frac{1}{\hbar \ell_{\mathrm{P}}^{2}} \varepsilon^{\mu \rho \sigma} e_{\rho}^{I} e_{\sigma}^{J} B_{\mu}^{a} .
$$

\begin{tabular}{|c|c|c|c|}
\hline $\begin{array}{c}\text { GR } \\
\text { smooth variable }\end{array}$ & $\begin{array}{c}\text { GR } \\
\text { discrete variable }\end{array}$ & $\begin{array}{c}\text { YM } \\
\text { smooth variable }\end{array}$ & $\begin{array}{c}\text { YM } \\
\text { discrete variable }\end{array}$ \\
\hline $\begin{array}{c}\operatorname{triad} e_{\mu}^{I} \\
\text { connection } \omega^{I J}{ }_{\mu}\end{array}$ & $\begin{array}{l}X_{s}^{I} \in \mathfrak{s} u(2) \\
g_{e} \in \mathrm{SU}(2)\end{array}$ & $\begin{array}{c}\text { auxiliary field } B_{\mu}^{a} \\
\text { connection } A_{\mu}^{a}\end{array}$ & $\begin{array}{l}B_{s}^{a} \in \mathfrak{g} \\
U_{e} \in G\end{array}$ \\
\hline
\end{tabular}

Squaring this and then symmetrizing over the four points, we obtain (12).

We summarize the discretisation of the dynamical variables in the following box.

Let us add two remarks before proceeding with the quantization.

- We have chosen to discretise both the gravitational and the YM connection on the dual triangulations. An alternative procedure, already considered in the literature [13], is to discretise the YM connection on the segments of the original triangulation $\Delta$, namely as $A_{\mu}^{a} \ell_{s}^{\mu}$ as opposed to $A_{\mu}^{a} \ell_{e}^{\mu}$ used in (18). Since the gravity sector provides the lengths $\ell_{s}$ of $\Delta$, this procedure has the advantage that the geometric information for the YM sector is directly described. The resulting spinfoam model presents however an awkward feature: the degrees of freedom of YM and GR are attached to different simplices. This obstructs a clear connection with the canonical formalism. The discretization proposed here, on the other hand, leads to a homogeneous description, as it will become clear below.

- The YM holonomy $U_{t}$ enters only the topological part of YM: the interaction term does not contain the group elements representing the gravitational and YM holonomies, but only the algebra elements $X_{s}^{I}$ and $B_{s}^{a}$. This is a consequence of using the action (7) to describe the coupled system that will be useful to define the spinfoam partition function.

\section{Spinfoams and quantisation of BF theory}

The quantum theory of (5) can be constructed from the spinfoam partition function

$$
Z_{\mathrm{PR}}=\prod_{t} \int_{\mathrm{SU}(2)} d g_{t} \prod_{s} \int_{\mathfrak{s} u(2)} d X_{s} e^{i \sum_{s} \operatorname{Tr}\left[X_{s} Z_{s}\right]}
$$

This quantity can be evaluated using the harmonic analysis of $\mathrm{SU}(2)$ (for details, see for instance $[1,9]$ ), and one obtains

$$
Z_{\mathrm{PR}}=\sum_{j_{s}} \prod_{s} \operatorname{dim} j_{s} \prod_{\tau}\{6 j\}
$$


where the sum is over all possible assignments of half-integers $j$ to the segments of $\Delta$. The half-integers, or spins, label the irreducible representations of $\mathrm{SU}(2)$. The quantity $\operatorname{dim} j \equiv 2 j+1$ is the dimension of the representation $j$. Finally, a $\{6 j\}$ symbol is associated with each tetrahedron $\tau$ of $\Delta$. The $\{6 j\}$ symbol is the key object of the recoupling theory of $\mathrm{SU}(2)$, and it depends only on the six $j \mathrm{~s}$ attached to the segments of the tetrahedron. It is defined in terms of the Wigner $3 \mathrm{~m}$ symbols (namely - up to normalization Clebsch-Gordan coefficients) as

$$
\left\{\begin{array}{ccc}
j_{1} & j_{2} & j_{3} \\
j_{4} & j_{5} & j_{6}
\end{array}\right\} \equiv\left(\begin{array}{ccc}
j_{1} & j_{2} & j_{3} \\
m_{1} & m_{2} & m_{3}
\end{array}\right)\left(\begin{array}{ccc}
j_{1} & j_{5} & j_{6} \\
m_{1} & m_{5} & m_{6}
\end{array}\right)\left(\begin{array}{ccc}
j_{4} & j_{5} & j_{3} \\
m_{4} & m_{5} & m_{3}
\end{array}\right)\left(\begin{array}{ccc}
j_{4} & j_{2} & j_{6} \\
m_{4} & m_{2} & m_{6}
\end{array}\right),
$$

where the sums over the repeated $m_{i}$ 's are understood. (22) defines the Ponzano-Regge model for riemannian $3 \mathrm{~d}$ quantum gravity in the absence of matter.

To quantize the coupled system, notice that in (7) there is also a second BF action, corresponding to the topological term of the YM action (6). For SU(2) YM theory, the quantization of this action is the same as in the PR model, only the interpretation of the variables differ. For $\mathcal{G}=\mathrm{SU}(N)$ with $N>2$ the quantization goes along the same lines described above, but the different algebraic properties of the group influence the final expression. In particular in (23), one uses a peculiarity of $\mathrm{SU}(2)$, namely the uniqueness of the Clebsch-Gordan decomposition. The tensor product of two $\mathrm{SU}(2)$ irreps is reducible into a direct sum of irreps, by means of the formula $j_{1} \otimes j_{2}=\left|j_{1}-j_{2}\right| \oplus \ldots \oplus j_{1}+j_{2}$. The peculiarity here is that each irrep in the RHS appears only once. ${ }^{4}$ This uniqueness is lost for $\mathrm{SU}(N)$ with $N>2$. Consider for instance $\mathrm{SU}(3)$, and let us use the Cartan highest weight notation to label the irreps; the tensor product of the two fundamental representations $(1,0) \otimes(0,1)$, contains twice the adjoint irrep $(1,1)$. As a consequence, the six labels on the segments are not enough to completely characterize a gauge invariant state. The additional numbers required are called intertwiners, and they are carried by the $3 \mathrm{~m}$ symbols. In the $\mathrm{SU}(3)$ example above, they distinguish between the two adjoints $(1,1)$, which have the same irrep labels. Recalling that combinatorially the $3 \mathrm{~m}$ symbols are associated to triangles, it should be clear that this additional data can be used to label the triangles of $\Delta$. Let us indicate by $\left\{i_{t}\right\}$ a collection of intertwiners associated with the triangles $\Delta$. Call $\rho$ the label for the irreps of $\mathrm{SU}(N)$; the partition function (22) generalizes for $\mathcal{G}=\mathrm{SU}(N)$ to

$$
Z_{\mathrm{BF}}[\mathcal{G}]=\sum_{\left\{\rho_{s}, i_{t}\right\}} \prod_{s} \operatorname{dim} \rho_{s} \prod_{\tau} A_{\tau}\left(\rho_{s}, i_{t}\right) .
$$

Only the labels for the segments and triangles belonging to $\tau$ enter the tetrahedron amplitude $A_{\tau}\left(\rho_{s}, i_{t}\right)$. When $N=2$, the tetrahedron amplitude reduces to the $\{6 j\}$ symbol described above. In the abelian case $\mathrm{U}(1)$, the tetrahedron amplitude is trivial; calling $n_{s}$ the irrep labels, we have only the combinatorial condition $\delta\left(\sum_{s \in t} n_{s}\right)$ for each triangle, which is the lattice equivalent of the Gauss law.

\subsection{Expectation values}

Geometric observables in the PR model are gauge-invariant functions of the variables $X_{s}^{I}$. To compute their expectation values it is convenient to introduce a generating functional,

$$
Z[J]=\prod_{t} \int_{\mathrm{SU}(2)} d g_{t} \prod_{s} \int_{\mathfrak{s} u(2)} d X_{s} e^{i \sum_{s} \operatorname{Tr} X_{s}\left(Z_{s}+J_{s}\right)} .
$$

This can be evaluated as described in [14], to give ${ }^{5}$

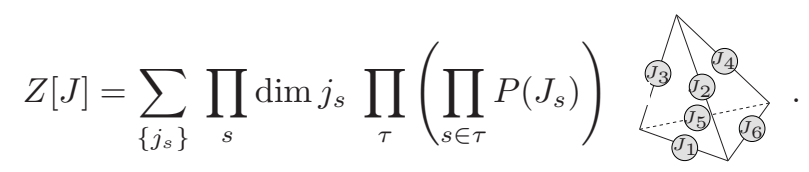

\footnotetext{
${ }^{4}$ The uniqueness of the $\mathrm{SU}(2)$ decomposition only concernes the $3 \mathrm{~d}$ case: for spacetime dimension $n>3$ there are generalized $3 n-j$ symbols appearing, which carry additional $n-3$ quantum numbers. Therefore, in dimension higher than 3 , we have intertwiners also for $\mathrm{SU}(2)$.

${ }^{5}$ To be more precise, the generating functional (25) is evaluated using a slightly different discretization procedure, in which the $X$-variables are not associated with the segments (which are dual to faces), but with the wedges, 2-surfaces introduced in [24] intersecting dual faces and triangles. For the applications of (25) in the rest of the paper, the wedge variables would give the same results as the $X_{s}$, thus for simplicity here we do not use them.
} 
The quantities entering this expression are defined as follows [14]. $P(J)$ is a function that relates the Lebesgue measure on $\mathbb{R}^{3}$ and the Haar measure on $\mathrm{SU}(2)$. Parametrizing as usual the algebra elements as $\vec{J}=\psi \hat{u}$, with $\hat{u}$ a unit vector on the 2 -sphere, we have $|J|=\psi$ and

$$
P(J)=\frac{2}{|J|} \sin \frac{|J|}{2} .
$$

The $\{6 j\}$ symbols with source insertions is given by

$$
\begin{aligned}
& \left(\begin{array}{ccc}
j_{1} & j_{2} & j_{3} \\
m_{1} & m_{2} & m_{3}
\end{array}\right)\left(\begin{array}{ccc}
j_{1} & j_{5} & j_{6} \\
n_{1} & m_{5} & m_{6}
\end{array}\right)\left(\begin{array}{ccc}
j_{4} & j_{5} & j_{3} \\
m_{4} & n_{5} & n_{3}
\end{array}\right)\left(\begin{array}{ccc}
j_{4} & j_{2} & j_{6} \\
n_{4} & n_{2} & n_{6}
\end{array}\right) \times \\
& D_{m_{1} n_{1}}^{\left(j_{1}\right)}\left(e^{J_{1}}\right) D_{m_{2} n_{2}}^{\left(j_{2}\right)}\left(e^{J_{2}}\right) D_{m_{3} n_{3}}^{\left(j_{3}\right)}\left(e^{J_{3}}\right) D_{m_{4} n_{4}}^{\left(j_{4}\right)}\left(e^{J_{4}}\right) D_{m_{5} n_{5}}^{\left(j_{5}\right)}\left(e^{J_{5}}\right) D_{m_{6} n_{6}}^{\left(j_{6}\right)}\left(e^{J_{6}}\right),
\end{aligned}
$$

where the $D$ 's are representation matrices. The $J$ 's are attached to the segments of $\Delta$; they are the sources of the quantum excitations $j_{s}$. If $J_{s}=0$ for all segments, (28) reduces to the expression for the $\{6 j\}$ symbol given above and $P(0)=1$, thus $\left.Z[J]\right|_{J=0} \equiv Z_{\mathrm{PR}}$.

Using the generating functional the expectation value of a gauge-invariant observable $\Phi\left[X_{s}^{I}\right]$ can be written as

$$
\langle\Phi\rangle=\prod_{t} \int_{\mathrm{SU}(2)} d g_{t} \prod_{s} \int_{\mathfrak{s} u(2)} d X_{s} \Phi\left[X_{s}^{I}\right] e^{i \sum_{s} X_{s}^{I} g_{s}^{I}}=\left.\Phi\left[-i \frac{\delta}{\delta J_{s}^{I}}\right] Z[J]\right|_{J=0} .
$$

To evaluate expressions of this type we need to know the action of the algebra derivatives on the measure term (27) and on the $\{6 j\}$ with sources (28). To evaluate the first action, notice that odd derivatives of $P(J)$ always vanish in $J=0$, and

$$
\left.\frac{\partial^{(2 n)}}{\partial J^{I_{1}} \ldots \partial J^{I_{2 n}}} P(J)\right|_{J=0}=\frac{(-1)^{n}}{2^{2 n}(2 n+1)} S^{I_{1} \ldots I_{2 n}},
$$

where $S^{I_{1} \ldots I_{2 n}}$ is the fully symmetrized tensor, namely $\delta^{I J}$ for $n=1,\left(\delta^{I J} \delta^{K L}+\delta^{I K} \delta^{J L}+\delta^{I L} \delta^{J K}\right) / 3$ for $n=2$, and so on. These derivatives only produce constant shifts, independent of the irrep label, and thus negligible at leading order in the large spin limit. The second action can be evaluated noticing that acting on a group element in the representation $j$, we have

$$
\left.\frac{\delta}{\delta J^{I}} D^{(j)}\left(e^{J}\right)\right|_{J=0}=-i T^{I(j)},
$$

where $T^{I(j)}$ is the $I$-th generator in the representation $j$. By inspecting (28), we see that a derivative acting on $J_{s}$ attaches to the segment $s$ an algebra generator in the irrep $j_{s}$ labeling the segment. This action is called "grasping", and it is common in the spinfoam literature (see for instance $[25,14,8]$ ). In particular, to study (11) we need the action of the double grasping, which enters twice (12), and the triple grasping, which enters the cubic term (13).

Let us introduce the following diagrammatic notation,

$$
-\frac{\delta}{\delta J_{s_{1}}^{I}} \frac{\delta}{\delta J_{s_{2}}^{I}} \equiv \underbrace{s_{1}}_{s_{2}}, \quad \mathcal{V}_{\tau}\left[-i \frac{\delta}{\delta J}\right]=\frac{1}{4} \sum_{p \in \tau} \frac{i}{3 !} \varepsilon_{I J K} \frac{\delta}{\delta J_{1}^{I}} \frac{\delta}{\delta J_{2}^{J}} \frac{\delta}{\delta J_{3}^{K}} \equiv,
$$

Notice that to shorten the notation, in the second diagram we included the sum, which we recall is over the four points in the tetrahedron, and 123 is a right-handed triple for each point. Concerning the double grasping, below we will need the cases when $s_{1}$ and $s_{2}$ coincide or share a point. In these two elementary cases the graspings give respectively $(\text { see }[8])^{6}$

$$
\}_{j}=\left.\left(C^{2}(j)+\frac{1}{4}\right)\right|_{j}, \quad j_{j_{1}}^{j_{2}} \succ_{j_{3}}=\frac{1}{2}\left[C^{2}\left(j_{3}\right)-C^{2}\left(j_{1}\right)-C^{2}\left(j_{2}\right)\right]_{j_{1}}^{j_{2}} \searrow_{j_{3}},
$$

\footnotetext{
${ }^{6}$ The shift $\frac{1}{4}$ in the double grasping on the same segment was omitted in [8], where we were mainly interested in studying the leading order of the semiclassical limit, to which it does not contribute. However notice that this term authomatically leads to the Ponzano-Regge ansatz $\ell^{2}=\left(j+\frac{1}{2}\right)^{2} \equiv j(j+1)+\frac{1}{4}$. I thank Etera Livine for pointing out to me this additional contribution.
} 
where $C^{2}(j)=j(j+1)$ is the $\mathrm{SU}(2)$ Casimir. We do not report the rather lengthy result for the triple grasping, for which we refer to [8]. Notice that the only contribution from (30) to these graspings is the shift of $1 / 4$ to the double grasping on the same segment. For the expectation values on a single tetrahedron $\tau$, we introduce also the following notation,

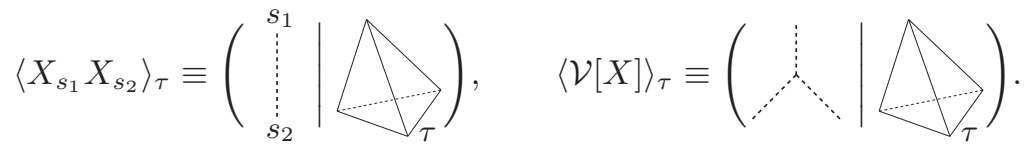

We proceed in the same way for the YM sector, introducing a source $\eta_{s}^{a}$ for the $B_{s}^{a}$ field and evaluating the generating functional as before, keeping in mind that for $\mathcal{G}=\mathrm{SU}(N)$ the partition function for $\mathrm{BF}$ depends also on additional quantum numbers labeling triangles, the intertwiners. Defining the $\mathrm{SU}(N)$ equivalent of (28) (where the intertwiners label the $3 \mathrm{~m}$ symbols), we have

$$
Z(\eta) \equiv \prod_{t} \int_{\mathcal{G}} d U_{t} \prod_{s} \int_{\mathfrak{g}} d B_{s} e^{i \sum_{s}\left[\operatorname{Tr} B_{s} W_{s}+\operatorname{Tr} B_{s} \eta_{s}\right]}=\sum_{\left\{\rho_{s}, i_{t}\right\}} \prod_{s} \operatorname{dim} \rho_{s} \prod_{\tau}\left(\prod_{s \in \tau} P\left(\eta_{s}\right)\right) \underset{\substack{\eta_{3} \\ \eta_{2} \eta_{5} \\ \eta_{3}}}{\eta_{\eta_{6}}} .
$$

To distinguish the GR graspings from the YM ones, we picture the latter with curly lines, such as

$$
-\frac{\delta}{\delta \eta_{s_{1}}^{I}} \frac{\delta}{\delta \eta_{s_{2}}^{I}} \equiv \sum_{s_{2}}^{s_{1}}
$$

As above, we have the elementary graspings

$$
\left.\left.\xi\right|_{\rho}=\left.\left(C^{2}(\rho)+\frac{1}{4}\right)\right|_{\rho}, \quad \rho_{\rho_{1}}^{\rho_{2}} \xi_{i \rho_{3}}=f\left(\rho_{i}, i\right) \sum_{\rho_{1}}^{\rho_{2}}\right\rangle_{i \rho_{3}} .
$$

Notice that now the non diagonal grasping depends also on the intertwiner label $i$, and $f$ depends on the gauge group considered. For $\mathcal{G}=\mathrm{SU}(2)$ there is no intertwiner and $f(\rho)=\frac{1}{2}\left[C^{2}\left(\rho_{3}\right)-C^{2}\left(\rho_{1}\right)-C^{2}\left(\rho_{2}\right)\right]$ as before.

Taking the product of (26) and (35) we define the generating functional for the two BF theories,

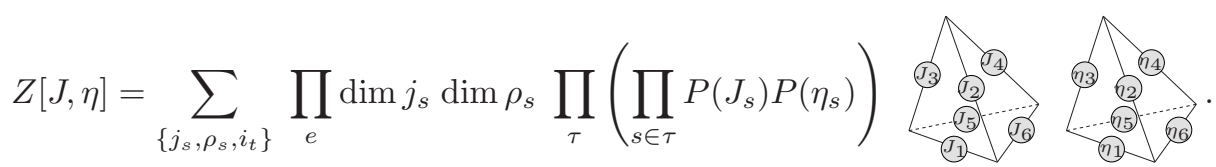

Using this generating functional we can compute the expectation values of functions of $X_{s}^{I}$ and $B_{s}^{a}$. In particular of (12) which gives the interaction between GR and YM. With the double graspings for GR and YM defined above the expectation value of (12) can be computed from the action of the following operator,

$$
\mathcal{C}_{\tau}\left[-i \frac{\delta}{\delta J},-i \frac{\delta}{\delta \eta}\right]=-\frac{1}{4} \sum_{p \in \tau} \sum_{s_{i}, t_{i} \in p} \varepsilon^{s_{1} s_{2} s_{3}} \varepsilon^{t_{1} t_{2} t_{3}} \frac{\delta}{\delta J_{s_{1}}^{I}} \frac{\delta}{\delta J_{t_{1}}^{I}} \frac{\delta}{\delta J_{s_{2}}^{J}} \frac{\delta}{\delta J_{t_{2}}^{J}} \frac{\delta}{\delta \eta_{s_{3}}^{a}} \frac{\delta}{\delta \eta_{t_{3}}^{a}}=\{,
$$

where the diagrammatic notation implicitly includes the sums, so the lines represent all the possible graspings within the tetrahedron. However the action of this operator on (35) is ambiguous, as it contains the product of two GR graspings: for non abelian groups the graspings do not commute, so to properly define this operator an ordering prescription is needed. We do so in the next section, motivated by recovering the right semiclassical limit. In section 6 below we come back to this generating functional and construct the spinfoam model for the coupled system.

\section{The semiclassical limit}

The spinfoam model that we are going to construct is defined on the fixed triangulation of spacetime introduced in section 3. Therefore we do not expect its semiclassical limit to reproduce the continuum action (2), but a suitable discretization of it. For the Ponzano-Regge model (22) this is well understood: in the limit when all the spins are large the discrete approximation to GR described by Regge calculus emerges. In this section we briefly recall the way this happens, and use it to fix the ordering prescription needed to deal with products of graspings. 


\subsection{Regge calculus}

It is an old result [3] that on a triangulation $\Delta$, made of flat tetrahedra, the curvature is distributional and concentrated on the segments. All the geometric information is encoded in the segment lengths $\ell_{s}$. The action can be written in terms of the segment lengths and their deficit angles $\varepsilon_{s}$,

$$
S\left[\ell_{s}\right]=\sum_{s \in \Delta} \ell_{s} \varepsilon_{s}\left(\ell_{s}\right) .
$$

The sum here is over all segments in $\Delta$. The dependence of the deficit angles on the segment lengths can be found as follows. First, the deficit angles are defined in terms of the dihedral angles as $\varepsilon_{s}=2 \pi-\sum_{\tau \ni s} \theta_{s}^{\tau}$. Then, the dihedral angles of a tetrahedron can be expressed in terms of the segments, through the well known formula

$$
\sin \theta_{s}=\frac{3}{2} \frac{\ell_{s} V}{A_{1} A_{2}}
$$

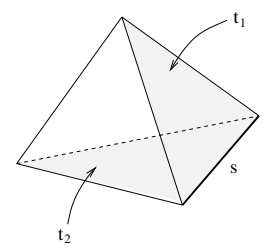

where $A_{1}$ and $A_{2}$ are the areas of the two triangles $t_{1}$ and $t_{2}$ sharing the segment $s$, as shown in the above figure. Finally the areas $A \equiv V_{(2)}$ and the volumes $V \equiv V_{(3)}$ can be expressed in terms of the segment lengths as determinants of the Cayley matrix,

$$
V_{(n)}^{2}=\frac{(-1)^{n+1}}{2^{n}(n !)^{2}} \operatorname{det} C_{(n)}, \quad C_{(n)}=\left(\begin{array}{ccccc}
0 & 1 & 1 & \ldots & 1 \\
1 & 0 & \ell_{1}^{2} & \ldots & \ell_{n}^{2} \\
1 & \ell_{1}^{2} & 0 & \ldots & \ell_{2 n-1}^{2} \\
\ldots & \ldots & \ldots & \ldots & \ldots \\
1 & \ell_{n}^{2} & \ell_{2 n-1}^{2} & \ldots & 0
\end{array}\right),
$$

A non trivial result of Regge calculus is that the deficit angles are the conjugate variables to the segment lengths,

$$
\varepsilon_{s}=\frac{\delta S}{\delta \ell_{s}} .
$$

Einstein's equations are read from $\delta S / \delta \ell_{s}=0$ on the bulk. From (43), we see that they imply a flat triangulation, with zero deficit angles everywhere in the bulk.

To see how this formalism for classical GR emerges from the PR model, we consider the Regge action on a single tetrahedron,

$$
S_{\mathrm{R}}\left[\ell_{s}\right]=\sum_{s \in \tau} \ell_{s} \theta_{s}\left(\ell_{s}\right) .
$$

The key fact is that exponentials of this action dominate the (homogeneous ${ }^{7}$ ) large spin limit of the $\{6 j\}$ symbol entering $(22)[5,7]$

$$
\{6 j\} \sim \frac{\cos \left(S_{\mathrm{R}}\left(j_{s}\right)+\frac{\pi}{4}\right)}{\sqrt{12 \pi V\left(j_{s}\right)}}
$$

where $V\left(j_{s}\right)$ and $S_{\mathrm{R}}\left(j_{s}\right)$ are respectively the classical volume (42) and the Regge action (44) of the tetrahedron with segment lengths given by $\ell=\ell_{\mathrm{P}}\left(j+\frac{1}{2}\right)$. The factor $\frac{\pi}{4}$ does not change the equations of motion, and the presence of the cosine as opposed to a single exponential is due to the fact that the PR model sums over both orientations of the tetrahedron, and the action (44) is odd under change of orientation (see discussions in $[1]) .^{8}$

If taking this limit is the correct way to study semiclassical physics, then also the geometric quantities that one can evaluate in the model should reduce to their classical expressions. Indeed, in [8] it was shown that the double and triple graspings reduce to classical scalar products and volumes. For the double grasping on two segments $i$ and $j$ sharing a point, we have the asymptotics

$$
(: \mid \bigwedge) \sim \ell_{s_{1}} \cdot \ell_{s_{2}} \frac{\cos \left(S_{\mathrm{R}}\left(j_{s}\right)+\frac{\pi}{4}\right)}{\sqrt{12 \pi V\left(j_{s}\right)}} .
$$

\footnotetext{
${ }^{7}$ Namely we rescale the half-integers entering the $\{6 j\}$ symbol as $j_{s} \equiv N k_{s}$ and take the $N \mapsto \infty$ limit. To keep a simple notation, we do not write explicitly this rescaling but keep using the variables $j_{s}$.

${ }^{8}$ This feature will also complicate the analysis of the coupled model as we discuss below.
} 
The scalar product here is defined in terms of the segment lengths in the natural way: if $\ell_{s_{3}}$ denotes the third segment in the triangle defined by $\ell_{s_{1}}$ and $\ell_{s_{2}}$, we have $2 \ell_{s_{1}} \cdot \ell_{s_{2}} \equiv \ell_{s_{3}}^{2}-\ell_{s_{1}}^{2}-\ell_{s_{2}}^{2}$. For the triple grasping, we have identically zero if the three segments are coplanar, and in any other case we get

$$
(\checkmark \mid \bigwedge j) \sim i V\left(j_{s}\right) \frac{\cos \left(S_{\mathrm{R}}\left(j_{s}\right)+\frac{3}{4} \pi\right)}{\sqrt{12 \pi V\left(j_{s}\right)}} .
$$

Notice that the volume is purely imaginary, and it has a different phase in the argument of the cosine. As described in [8] and anticipated in [14], this is due to the fact that the PR model sums over both orientations of spacetime, and the definition (13) of volume changes sign under change of orientation. ${ }^{9}$ Consequently, real results with the correct phase can be obtained looking at even powers.

As the graspings do not commute with each other, ordering ambiguites arise in defining the products of graspings. As discussed in [8], we can choose a "temporal ordering" $T$ prescribing the graspings to be performed one after the other. Under this prescription we have

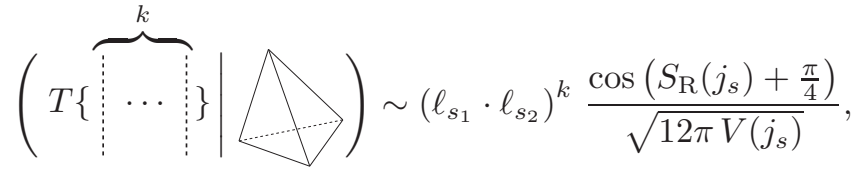

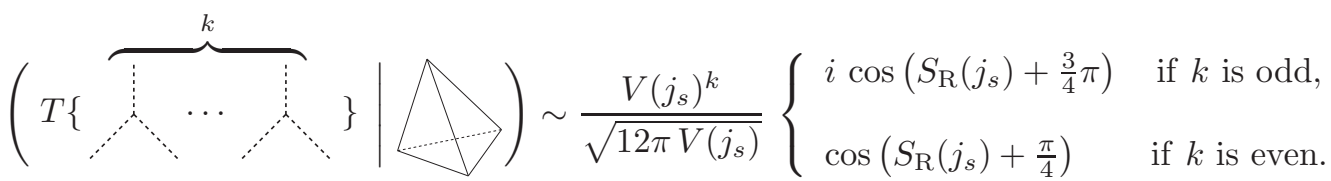

This is the way Regge calculus emerges from spinfoams in the large spin limit: the partition function reduces to exponential of the Regge action, and the expectation values of the geometry to their classical counterparts. This means that the theory has a well defined semiclassical limit. Below we are going to construct a spinfoam model for the coupled system of GR and YM. If this framework is indeed robust, then the coupled system should have a semiclassical limit, given by large irrep labels, in which gravity is described by Regge calculus and YM theory by a discretization à la Regge, which we construct below.

\subsection{Regge discretization of YM theory}

We discuss here how (6) can be written in the Regge approach introduced above. First of all, we consider the topological BF term. To keep things explicit, let us focus on the special case $\mathcal{G}=\mathrm{SU}(2)$. By analogy with (45), we know that the large $\rho$ limit of (24) will be dominated by the cosine of the discrete action $S=\sum_{s} B_{s} \psi_{s}\left(B_{s}\right)$ where $B_{s}=\hbar\left(\rho_{s}+\frac{1}{2}\right)$ represents lengths in the fibre space. The "dihedral angles" $\psi_{s}$ measure the curvature of the fibre space, and the equations of motion impose it flat. This is just a straighforward discrete version of the BF action. More interesting is the Regge description of the term (8) which makes YM dynamical and carries the interaction between GR and YM. We discretize this by a sum over flat tetrahedra as

$$
\frac{g_{0}^{2}}{2} \int d^{3} x \sqrt{g} g^{\mu \nu} B_{\mu}^{a} B_{\nu}^{a} \sim 3 g_{0}^{2} \sum_{\tau} V_{\tau} g^{\mu \nu} B_{\mu}^{a} B_{\nu}^{a},
$$

where we have replaced the canonical volume form $d^{3} x \sqrt{g}$ by (six times) the tetrahedron volume $V_{\tau}$. To express (50) in terms of Regge variables, we need the flat inverse metric as a function of the segment lengths. To obtain this expression, we we start from the formula for the volume $V$ of the tetrahedron,

$$
(3 ! V)^{2}=g=\frac{1}{3 !} \varepsilon^{\mu_{1} \ldots \mu_{n}} \varepsilon^{\nu_{1} \ldots \nu_{n}} g_{\mu_{1} \nu_{1}} \cdots g_{\mu_{n} \nu_{n}} .
$$

Deriving this, we obtain

$$
(3 !)^{2} \frac{\partial V^{2}}{\partial g_{\mu \nu}}=\frac{3}{3 !} \varepsilon^{\mu \mu_{2} \ldots \mu_{n}} \varepsilon^{\nu \nu_{2} \ldots \nu_{n}} g_{\mu_{2} \nu_{2}} \cdots g_{\mu_{n} \nu_{n}}=(3 !)^{2} V^{2} g^{\mu \nu} .
$$

\footnotetext{
${ }^{9}$ This could be avoided introducing a modulus in the definition of the volume, as is done in the canonical approach [25]. However, this would prevent a definition of the functional derivatives in terms of graspings.
} 
Expressing the derivative in terms of the squared edge lengths $\ell_{s}^{2}=g_{\mu \nu} \ell_{s}^{\mu} \ell_{s}^{\nu}$, we read

$$
g^{\mu \nu}=\frac{2}{V} \frac{\partial V}{\partial g_{\mu \nu}}=\frac{2}{V} \sum_{s \in \tau} \frac{\partial V}{\partial \ell_{s}^{2}} \frac{\partial \ell_{s}^{2}}{\partial g_{\mu \nu}}=\frac{2}{V} \sum_{s \in \tau} \frac{\partial V}{\partial \ell_{s}^{2}} \ell_{s}^{\mu} \ell_{s}^{\nu}
$$

where the sum is over the six segments in the tetrahedron.

Using (53) and (17), we can write (50) as

$$
S_{\mathrm{RInt}}\left[\ell_{s}, B_{s}\right]=3 ! \hbar^{2} g_{0}^{2} \sum_{\tau} \sum_{s \in \tau} \frac{\partial V_{\tau}}{\partial \ell_{s}^{2}} B_{s}^{a} B_{s}^{a}
$$

To compute the volume derivatives, it is convenient to introduce a double index notation, where a segment is identified by its vertices, $s \equiv i j$ with $i, j=1 \ldots 4$. Let us also introduce the vectors $\vec{n}_{i}$ normal to the triangle obtained removing the point $i$ from the tetrahedron. We choose their orientation so that they point inward. These vectors satisfy $\left|\vec{n}_{i}\right|^{2}=4 A_{i}^{2}, \vec{n}_{i} \cdot \vec{n}_{j}=4 A_{i} A_{j} \cos \theta_{i j}$, where $\theta_{i j}$ are the dihedral angles defined in (41). Then, deriving (42) for $n=3$ and using (41) we obtain

$$
\frac{\partial V}{\partial \ell_{i j}^{2}}=\frac{1}{72} \frac{\vec{n}_{i} \cdot \vec{n}_{j}}{V}
$$

In conclusion, we can write the interaction term on a Regge triangulation as

$$
S_{\mathrm{RInt}}\left[\ell_{s}, B_{s}\right]=\frac{1}{12} \hbar^{2} g_{0}^{2} \sum_{\tau} \sum_{i j \in \tau} \frac{\vec{n}_{i} \cdot \vec{n}_{j}}{V_{\tau}} B_{i j}^{a} B_{i j}^{a} .
$$

We have defined the ordering prescription to deal with the products of graspings, and described the Regge description of the classical coupled system. Below we will show that this is exactly what emerges in the semiclassical limit of the quantum theory.

\section{Spinfoam model of the coupled system}

We now come back to the construction of the spinfoam model for the coupled system. To define the quantum theory for the coupled system, we evaluate the partition function

$$
Z_{\mathrm{GRYM}}=\int \mathcal{D} e \mathcal{D} \omega \mathcal{D} B \mathcal{D} A e^{\frac{i}{\ell_{\mathrm{P}}} \int \operatorname{Tr} e \wedge F(\omega)+\frac{i}{\hbar} \int \operatorname{Tr} B \wedge F(A)-\frac{i}{\hbar} \frac{g_{0}{ }^{2}}{2} \int \operatorname{Tr} B \wedge * B} .
$$

Firstly, we regularize this formal expression by means of the discretization procedure discussed above, obtaining

$$
Z_{\mathrm{GRYM}}=\prod_{t} \int_{S U(2)} d g_{t} \prod_{t} \int_{\mathcal{G}} d U_{t} \prod_{s} \int_{\mathfrak{s} u(2)} d X_{s} \prod_{s} \int_{\mathfrak{g}} d B_{s} e^{i \sum_{s}\left[\operatorname{Tr} X_{s} Z_{s}+\operatorname{Tr} B_{s} W_{s}\right]-i \lambda S_{\mathrm{BB}}[X, B]},
$$

with $\lambda S_{\mathrm{BB}}$ defined in (11). We see explicitly from the expression above the advantage of the symmetric form of the action obtained using the first order formalism for YM theory. In fact, the interaction term $S_{\mathrm{BB}}[X, B]$ is a gauge invariant function of the algebra variables only, and does not contain gravitational nor YM holonomies. We can thus use the generating functional (38) and with the substitutions $X_{s}^{I} \mapsto-i \frac{\delta}{\delta J_{s}^{I}}$, $B_{s}^{a} \mapsto-i \frac{\delta}{\delta \eta_{s}^{a}}$, write

$$
Z_{\mathrm{GRYM}}=\left.e^{-i \lambda S_{\mathrm{BB}}\left[-i \frac{\delta}{\delta J},-i \frac{\delta}{\delta \eta}\right]} Z[J, \eta]\right|_{J=\eta=0}
$$

We can interpret the equation above in the following sense: $Z[J, \eta]$ gives a colouring of the triangulation, with labels for $\mathrm{SU}(2)$ and $\mathcal{G}$ irreps on the edges, and labels for $\mathcal{G}$ intertwiners on the triangles. This colouring provides the kinematical arena for the dynamics of YM and the interaction between YM theory and gravity, which is realized by the action of the local operator $e^{i \lambda S_{\mathrm{BB}}}$. 
To define $S_{\mathrm{BB}}\left[-i \frac{\delta}{\delta J},-i \frac{\delta}{\delta \eta}\right]$, we use the $T$-ordering introduced above, but we also need a prescription for the inverse volume $(\mathcal{V}[X])^{-1}$ appearing in (11). Motivated by recovering the right semiclassical limit, we define this operator as

$$
\left(\mathcal{V}_{\tau}\left[-i \frac{\delta}{\delta J}\right]\right)^{-1} \equiv \int_{0}^{\infty} d t \sum_{k=0}^{\infty} \frac{(-t)^{k}}{k !}\left(\mathcal{V}_{\tau}\left[-i \frac{\delta}{\delta J}\right]\right)^{2 k+1}
$$

where $t$ is an auxiliary variable with no physical meaning, ${ }^{10}$ and the powers of the triple grasping are $T$ ordered as in (49). The advantage of this definition is that, using (49), we have in the large spin limit the desired semiclassical behaviour of the inverse volume,

$$
\left\langle\left(\mathcal{V}_{\tau}\left[-i \frac{\delta}{\delta J}\right]\right)^{-1}\right\rangle \sim i \frac{1}{V\left(j_{s}\right)} \frac{\cos \left(S_{\mathrm{R}}\left(j_{s}\right)+\frac{3}{4} \pi\right)}{\sqrt{12 \pi V\left(j_{s}\right)}}
$$

With these definitions, we can evaluate the partition function as a power series in $\lambda$ :

$$
\left.Z_{\mathrm{GRYM}} \equiv \sum_{n} \frac{(-i \lambda)^{n}}{n !}\left(S_{\mathrm{BB}}\left[-i \frac{\delta}{\delta J},-i \frac{\delta}{\delta \eta}\right]\right)^{n} Z[J, \eta]\right|_{J=\eta=0},
$$

where

$$
S_{\mathrm{BB}}\left[-i \frac{\delta}{\delta J},-i \frac{\delta}{\delta \eta}\right]=\sum_{\tau} \int_{0}^{\infty} d t \sum_{k=0}^{\infty} \frac{(-t)^{k}}{k !}\left(V_{\tau}\left[-i \frac{\delta}{\delta J}\right]\right)^{2 k+1} \mathcal{C}_{\tau}\left[-i \frac{\delta}{\delta J},-i \frac{\delta}{\delta \eta}\right]
$$

The expression (61) provides us with a formula for the partition function (57) in the spinfoam formalism. It is well defined, order by order in $\lambda$. The ordering prescription for the operators is as in (48) and (49). $S_{\mathrm{BB}}$ is local because the graspings only connect segments belonging to the same tetrahedron. Notice that this partition function provides a quantization of YM theory only when the full series is considered. As discussed in [21], the order-by-order equivalence requires additional gauge-fixing terms, which change the topological nature of the zeroth order and thus the spinfoam procedure used here.

The zeroth order of the coupled partition function (61) corresponds to the product of two independent BF partition functions,

$$
\left.Z^{(0)} \equiv Z[J, \eta]\right|_{J=\eta=0}=Z_{\mathrm{BF}}[S U(2)] Z_{\mathrm{BF}}[\mathcal{G}] .
$$

It is a sum over all the labelings of all the segments of the triangulation by irreps of $\mathrm{SU}(2)$ and $\mathcal{G}$, where the $\mathrm{SU}(2)$ labels describe the geometry of the triangulation, as in the pure gravity picture, and the $\mathcal{G}$ labels describe the degrees of freedom of YM theory. We report in the following table the homogeneity of the labeling, which has been achieved discretizing both GR and YM connections on the dual triangulation:

\begin{tabular}{|l||c|c|c|c|c|}
\hline simplex & GR labels & YM labels & GR amplitude & YM amplitude & Interaction \\
point & & & & & \\
segment & $j$ & $\rho$ & $\operatorname{dim} j$ & $\operatorname{dim} \rho$ & \\
triangle & & $i$ & $\{6 j\}$ & $A_{\tau}(\rho, i)$ & $S_{\mathrm{BB}}\left[j_{s}, \rho_{s}, i_{t}\right]$ \\
\hline
\end{tabular}

This structure allows us to identify $2 \mathrm{~d}$ slices of the spinfoam with a spin network state for the canonical quantization of the coupled system. The links of such a spin network would be coloured by the labels for the irreps of GR's $\mathrm{SU}(2)$ and YM's $\mathrm{SU}(N)$, and on the nodes by the intertwiners of $\mathrm{SU}(N):|s\rangle=\left|\gamma, j_{l}, \rho_{l}, i_{n}\right\rangle$.

In the next section we study the metric dependence of the interaction term $S_{\mathrm{BB}}\left[j_{s}, \rho_{s}, i_{t}\right]$.

\footnotetext{
${ }^{10}$ If we compare it with the Turaev-Viro model [26], we see that $t$ behaves like the contribution, from a single tetrahedron, to an imaginary cosmological constant, $\Lambda=i t$.
} 


\subsection{Effective action}

The first order term in the expansion $(61)$ is $Z^{(1)} \equiv-i \lambda\left\langle S_{\mathrm{BB}}\right\rangle$, thus it amounts to computing the effective action for the interacting term (8). For the $\mathrm{SU}(2)$ graspings we use the diagrammatic notation (32), and we $T$-order them as in (48), (49). The double grasping on $\mathcal{G}$ has no ordering ambiguities (it appears only linearly at this order, and it commutes with the other graspings). Introducing the diagrammatic notation as in (32), we can write

$$
\begin{aligned}
& \left\langle S_{\mathrm{BB}}\right\rangle=\sum_{\left\{j_{s}, \rho_{s}, i_{t}\right\}} \int_{0}^{\infty} d t \prod_{s} \operatorname{dim} j_{s} \operatorname{dim} \rho_{s} \sum_{\tilde{\tau}} \prod_{\tau \neq \tilde{\tau}}\{6 j\} A_{\tau}\left(\rho_{s}, i_{t}\right)\left[\left(T \{ \begin{array} { c } 
{ \vdots } \\
{ \vdots } \\
{ \vdots }
\end{array} \} \left\{\mid\left\langle\bigwedge_{\tilde{\tau}}\right)+\right.\right.\right.
\end{aligned}
$$

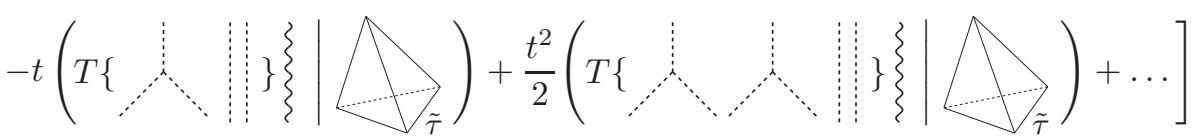

The key diagram to evaluate is

$$
\left\langle\mathcal{C}_{\tau}\right\rangle=\left(\begin{array}{l:l}
T\{ & \} \\
& \left\{\bigwedge_{\tau}\right.
\end{array}\right) .
$$

This diagram has $4 \times 36=144$ contributions, coming from all the possible choices of graspings in a given point, times the four points. Each contribution can be evaluated using grasping rules and recoupling theory as in [8]. Because there are only double graspings entering this expression, the evaluation is rather simple and we do not report the details here, but only the asymptotics.

Let us distinguish two types of terms, when the YM grasping is diagonal, namely $s_{3}=t_{3}$, and when is not diagonal, namely $s_{3} \neq t_{3}$. Consider first the diagonal case. For fixed $s_{3}=i j$, there are 4 contributions from $p=i$, and four from $p=j$. To fix ideas, let us choose $s_{3}=12$. The YM grasping immediately gives $C^{2}\left(\rho_{12}\right)$ from (37). As for the GR grasping, we can implement the antisymmetrization from the $\varepsilon$ tensors of (39) in the algebraic indeces, and using (48) the large spin limit of the relevant graspings from $p=1$ gives

$$
2\left\langle X_{13}^{I} X_{13}^{[I} X_{14}^{J} X_{14}^{J]}\right\rangle \sim \frac{\cos \left(S_{\mathrm{R}}\left(j_{s}\right)+\frac{\pi}{4}\right)}{\sqrt{12 \pi V\left(j_{s}\right)}} 2\left[\ell_{13}^{2} \ell_{14}^{2}-\left(\ell_{13} \cdot \ell_{14}\right)^{2}\right] .
$$

The term in square brackets can be immediately recognized as $4 A_{2}{ }^{2}$. Analogously, the graspings from $p=2$ give $4 A_{1}{ }^{2}$. There are in total 48 contributions of this type.

The second case is when the YM grasping is non diagonal. Let us fix $s_{3}=12, t_{3}=13$. Choosing for simplicity $\mathrm{SU}(2)$, the YM grasping gives $\frac{1}{2}\left[C^{2}\left(\rho_{23}\right)-C^{2}\left(\rho_{12}\right)-C^{2}\left(\rho_{13}\right)\right]$. The GR graspings have four contributions all coming from $p=1$, times 2 from the symmetric choice $s_{3}=13, t_{3}=12$, giving in total the eight contributions

$$
-4\left\langle X_{13}^{I} X_{12}^{[I} X_{14}^{J} X_{14}^{J]}\right\rangle \sim \frac{\cos \left(S_{\mathrm{R}}\left(j_{s}\right)+\frac{\pi}{4}\right)}{\sqrt{12 \pi V\left(j_{s}\right)}} 4\left[\left(\ell_{12} \cdot \ell_{14}\right)\left(\ell_{13} \cdot \ell_{14}\right)-\left(\ell_{12} \cdot \ell_{13}\right) \ell_{14}^{2}\right] .
$$

If we recall the definition of the dihedral angles $\theta_{s}$ in terms of angles $\phi_{i j}$ between the segment vectors (defined by $\left.\ell_{i j} \cdot \ell_{i k}=\ell_{i j} \ell_{i k} \cos \phi_{j k}\right)$,

$$
\sin \phi_{i j} \sin \phi_{j k} \cos \theta_{i k}=\cos \phi_{i j} \cos \phi_{j k}-\cos \phi_{i k},
$$

the term in square brackets in (66) reads $\vec{n}_{2} \cdot \vec{n}_{3}$. Notice that the same contribution comes also from the choice $s_{3}=24, t_{3}=34$, for which the YM grasping gives $\frac{1}{2}\left[C^{2}\left(\rho_{23}\right)-C^{2}\left(\rho_{24}\right)-C^{2}\left(\rho_{34}\right)\right]$. There are in total 96 contributions of this type.

Adding up all the contributions we get

$$
\begin{aligned}
& 8 C^{2}\left(\rho_{12}\right)\left(A_{1}^{2}+A_{2}^{2}\right)+\ldots \\
& -4 \vec{n}_{2} \cdot \vec{n}_{3}\left[\frac{1}{2}\left(C^{2}\left(\rho_{23}\right)-C^{2}\left(\rho_{12}\right)-C^{2}\left(\rho_{13}\right)\right)+\frac{1}{2}\left(C^{2}\left(\rho_{23}\right)-C^{2}\left(\rho_{24}\right)-C^{2}\left(\rho_{34}\right)\right)\right]+\ldots
\end{aligned}
$$


Collecting all the Casimirs and using the fact that $\sum_{i=1}^{4} \vec{n}_{i} \equiv 0,(68)$ adds up to $-8 \sum_{i j} \vec{n}_{i} \cdot \vec{n}_{j} C^{2}\left(\rho_{i j}\right)$. Therefore (64) has the following large spin behaviour,

$$
\left\langle\mathcal{C}_{\tau}\right\rangle \sim-2 \sum_{i j} \vec{n}_{i} \cdot \vec{n}_{j} C^{2}\left(\rho_{i j}\right) \frac{\cos \left(S_{\mathrm{R}}\left(j_{s}\right)+\frac{\pi}{4}\right)}{\sqrt{12 \pi V\left(j_{s}\right)}} \frac{\cos \left(S_{\mathrm{R}}\left(\rho_{s}\right)+\frac{\pi}{4}\right)}{\sqrt{12 \pi V\left(\rho_{s}\right)}}
$$

Next, we look at the other diagrams entering (63). Thanks to the $T$-ordering, the powers of the triple grasping factorize in the large spin limit and one obtains simply (60). Putting everything together and approximating the sums over the irrep labels with integrals, we then have

$$
\begin{aligned}
-i \lambda\left\langle S_{\mathrm{BB}}\right\rangle & \sim \int \prod_{s} d j_{s} d \rho_{s} \prod_{s} \operatorname{dim} j_{s} \operatorname{dim} \rho_{s} \sum_{\tilde{\tau}} \prod_{\tau \neq \tilde{\tau}}\{6 j\}\{6 \rho\} \times \\
& \times\left[\frac{g_{0}^{2}}{12} \hbar \ell_{\mathrm{P}} \sum_{i j} \frac{\vec{n}_{i} \cdot \vec{n}_{j}}{V_{\tilde{\tau}}} C^{2}\left(\rho_{i j}\right)\right] \frac{\cos \left(S_{\mathrm{R}}\left(j_{s}\right)+\frac{3}{4} \pi\right)}{\sqrt{12 \pi V\left(j_{s}\right)}} \frac{\cos \left(S_{\mathrm{R}}\left(\rho_{s}\right)+\frac{\pi}{4}\right)}{\sqrt{12 \pi V\left(\rho_{s}\right)}} .
\end{aligned}
$$

In the limit in which $C^{2}\left(\rho_{i j}\right) \sim B_{i j}^{a} B_{i j}^{a}$, the term in square brackets is exactly $\frac{1}{\hbar} S_{\text {RInt }}$ given in (56). This is our key result: the large spin behaviour of the spinfoam model (61) is dominated by the classical dynamics of GR coupled to YM described à la Regge.

\subsection{Tetrahedral asymptotics of the coupled partition function}

Let us now go back to the full partition function (61), and consider a single tetrahedron. In this simple case, we can proceed as above to show the general result for powers of the operator $S_{\mathrm{BB}}$,

$$
(-i \lambda)^{n}\left\langle S_{\mathrm{BB}}{ }^{n}\right\rangle \sim\left(i S_{\mathrm{RInt}}\right)^{n} \frac{\cos \left(S_{\mathrm{R}}\left(\rho_{s}\right)+\frac{\pi}{4}\right)}{\sqrt{12 \pi V\left(j_{s}\right)} \sqrt{12 \pi V\left(\rho_{s}\right)}} \begin{cases}i \cos \left(S_{\mathrm{R}}\left(j_{s}\right)+\frac{3}{4} \pi\right) & \text { if } n \text { is odd, } \\ \cos \left(S_{\mathrm{R}}\left(j_{s}\right)+\frac{\pi}{4}\right) & \text { if } n \text { is even. }\end{cases}
$$

Using (71) we can sum up the series (61) and evaluate the coupled partition function in the large spin limit,

$$
\begin{aligned}
Z & \sim \int \prod_{s} d j_{s} d \rho_{s} \prod_{s} \operatorname{dim} j_{s} \operatorname{dim} \rho_{s} \frac{\cos \left(S_{\mathrm{R}}\left(\rho_{s}\right)+\frac{\pi}{4}\right)}{\sqrt{12 \pi V\left(j_{s}\right)} \sqrt{12 \pi V\left(\rho_{s}\right)}} \times \\
& \times\left[\sum_{n=0}^{\infty} \frac{\left(i S_{\mathrm{RInt}}\right)^{2 n+1}}{(2 n+1) !} i \cos \left(S_{\mathrm{R}}\left(j_{s}\right)+\frac{3}{4} \pi\right)+\sum_{n=0}^{\infty} \frac{\left(i S_{\mathrm{RInt}}\right)^{2 n}}{(2 n) !} \cos \left(S_{\mathrm{R}}\left(j_{s}\right)+\frac{\pi}{4}\right)\right]= \\
& =\int \prod_{s} d j_{s} d \rho_{s} \prod_{s} \operatorname{dim} j_{s} \operatorname{dim} \rho_{s} \frac{\cos \left(S_{\mathrm{R}}\left(j_{s}\right)-S_{\mathrm{RInt}}\left(j_{s}, \rho_{s}\right)+\frac{\pi}{4}\right) \cos \left(S_{\mathrm{R}}\left(\rho_{s}\right)+\frac{\pi}{4}\right)}{\sqrt{12 \pi V\left(j_{s}\right)} \sqrt{12 \pi V\left(\rho_{s}\right)}} .
\end{aligned}
$$

We see from this result that the asymptotics of the tetrahedral partition function are dominated by linear combinations of exponentials of the classical discrete actions described in section 5. Notice that in principle one would expect a single exponential of the Regge version of (7) from a correct semiclassical limit, namely

$$
e^{-i\left[S_{\mathrm{R}}\left(j_{s}\right)+S_{\mathrm{R}}\left(\rho_{s}\right)-S_{\mathrm{RInt}}\left(j_{s}, \rho_{s}\right)\right]} .
$$

Yet the presence of the somewhat akward linear combination in (72) can be completely understood if we recall that the spinfoam quantization includes a sum over both orientation of the (triangulated) spacetime manifold. In fact, let us look at (7): under change of orientation $\left(e_{\mu}^{I} \mapsto-e_{\mu}^{I}\right)$ both the first and third terms change sign, but not the second. This accounts for the first cosine term in (72),

$$
\left(e^{i\left[S_{\mathrm{R}}\left(j_{s}\right)-S_{\mathrm{RInt}}\left(j_{s}, \rho_{s}\right)\right]}+e^{-i\left[S_{\mathrm{R}}\left(j_{s}\right)-S_{\mathrm{RInt}}\left(j_{s}, \rho_{s}\right)\right]}\right) e^{i S_{\mathrm{R}}\left(\rho_{s}\right)} .
$$

The second cosine comes naturally when the same argument is applied also to the fibre manifold: under change of orientation of the fibres $\left(B_{\mu}^{a} \mapsto-B_{\mu}^{a}\right)$ only the second term in (7) changes sign, whereas the first and third do not. 
So the tetrahedral partition function (72) is the correct semiclassical limit of a coupled quantum theory with the feature of summing over both orientations of the spacetime and fibre manifold. However it is exactly the presence of this sum over orientations that makes problematic the interpretation of the partition function on a generic triangulation, whose semiclassical limit we do not report here. In the PR model this is not a problem: the model is topological invariant, thus the partition function on a generic triangulation can be reduced to the one on a single tetrahedron. The model presented here on the other hand is manifestly non topological invariant, thus this argument does not apply. We leave this question open, stressing that it is an issue of the PR model in its own, and not of the coupling with YM performed here. Furthermore, notice that the problem can be circumvented by introducing oriented boundary states in the computation of physical correlations, as it is done in the graviton calculations of [2].

\section{Conclusions}

In this paper we defined a spinfoam model for riemannian 3d quantum gravity coupled to Yang-Mills theory. The partition function of this model is given in (61). The construction makes use of the generating functional technique, and of grasping rules and $\mathrm{SU}(2)$ recoupling thery. With respect to previous attempts in the literature, the model has the advantage of discretizing the gravitational and YM connections in the same way. This leads to a homogeneous labeling of the triangulation by the GR and YM variables, as reported in the table in section 6 , and thus this model provides transition amplitudes for spin networks of the canonical theory. Furthermore, for the particular choice of SU(2) YM theory, we were able to show explicitly that the model has the correct semiclassical limit, given by the discretization à la Regge of the classical YM action. This result supports both the consistency of coupling matter fields to gravity in the way proposed here, and the utility of the large spin limit to study semiclassical physics.

Let us add a few comments and perspectives on further developments. One thing to stress is that as usual, the quantization procedure presents many ambiguities. In particular, here we chose a definite way of discretizing the dynamical variables and the actions, and we gave a particular ordering prescription for the products of non commuting operators. Other choices are possible. This one was preferred because it easily reproduced the correct semiclassical limit. Once the correct limit is established, the key question becomes computing the quantum gravity corrections to it. Already in the simple model presented here we see that this is a formidable task due to the large number of contributions that the next to leading order of the asymptotics receives. Yet this crucial question certainly deserves further work. Among the intriguing effects to look for there are modified dispersion relations: even though the discrete structure of loop quantum gravity does not break Lorentz invariance per se [27], it is commonly recognized that possible deformations of this symmetry is a promising sector to look for observable quantum gravity effects [28]. Remarkably in [10] it was shown that the coupling of point particles to the Ponzano-Regge model gives a well defined non commutative effective action for the particles, thus resulting in a precise Lorentz deformation with modified dispersion relations. It would be extremely interesting if analogue effects arise in the model here presented. ${ }^{11}$ A potential difference lies in the fact that here the matter field is added to the gravitational action, whereas in the approach of [10] the particles are described using the gauge degrees of freedom of the gravitational sector. However the situation could be similar to what happens in quantum field theory in the temporal gauge, for both YM theory [29] and linearized gravity [30], where static matter sources (whose coupling is lost by the choice of gauge) turn out to be described by gauge degrees of freedom.

At the end of the previous section we mentioned how an oriented boundary state could fix the orientation of the coupled partition function. Indeed, finding a suitable boundary state would have far more important applications: it would allow to follow the proposal developed in [2] to compute scattering amplitudes for the coupled system described here. This would be a key development to understand low energy physics in the context of spinfoam gravity. From this point of view, the emergence of Regge calculus in the large spin limit is very promising, as this property is at the basis of the results in [2], and can be used to construct a suitable boundary state, as discussed in [31].

Another important question for future work is how to restore the full diffeomorphism invariance of the continuum theory, here broken by the choice of a fixed triangulation. Being the model non topological, the symmetry should be restored by including a sum over the triangulations, for instance along the lines of the group field theory approach [23]. Notice that restoring the right degrees of freedom can only be expected

\footnotetext{
${ }^{11}$ Notice that the quantum particle of $3 \mathrm{~d}$ YM theory is massless and spinless, thus the results of [10] might not apply straighforwardly.
} 
for the fully resummed partition function. This is because as pointed out in section 6 , only the full partition function can be expected to be equivalent to YM theory. This important aspect will be studied elsewhere.

Finally, it is sometimes argued that the spinfoam quantization of $\mathrm{SU}(2)$ BF theory simply amounts to mapping the $B$ field to its eigenvalue $j$. This map has been considered in the literature, especially in $4 \mathrm{~d}$, to define the spinfoam quantization of additional terms to the BF action, may they be constraints reducing $\mathrm{BF}$ to GR, or matter fields. We would like to stress that particular care should be taken in using this map: the more consistent procedure used in this paper, where the additional terms act as genuine quantum operators, shows that such a map would fail to capture the full quantum theory. In particular, one would not see the ordering ambiguities and would fail to reproduce the full richness of the quantum corrections to the semiclassical leading order.

\section{Acknowledgments}

The author is particularly grateful to Carlo Rovelli, Laurent Freidel, Hendryk Pfeiffer and John Barrett for many discussions and suggestions.

Research at Perimeter Institute for Theoretical Physics is supported in part by the Government of Canada through NSERC and by the Province of Ontario through MRI.

\section{References}

[1] C. Rovelli. Quantum Gravity. (Cambridge University Press, Cambridge 2004.)

[2] C. Rovelli, "Graviton propagator from background-independent quantum gravity," Phys. Rev. Lett. 97 (2006) 151301 [arXiv:gr-qc/0508124].

S. Speziale, "Towards the graviton from spinfoams: The 3d toy model," JHEP 05 (2006) 039 [arXiv:grqc/0512102].

E. Bianchi, L. Modesto, C. Rovelli and S. Speziale, "Graviton propagator in loop quantum gravity," Class. Quant. Grav. 23 (2006) 6989 [arXiv:gr-qc/0604044].

E. R. Livine, S. Speziale and J. L. Willis, "Towards the graviton from spinfoams: Higher order corrections in the 3d toy model," Phys. Rev. D 75 (2007) 024038 [arXiv:gr-qc/0605123].

E. R. Livine and S. Speziale, "Group integral techniques for the spinfoam graviton propagator," JHEP 0611 (2006) 092 [arXiv:gr-qc/0608131].

[3] T. Regge, "General relativity without coordinates," Nuovo Cim. 19 (1961) 558.

[4] G. Immirzi, "Quantum gravity and Regge calculus," Nucl. Phys. Proc. Suppl. 57 (1997) 65 [arXiv:grqc/9701052].

[5] G. Ponzano, T. Regge. "Semiclassical limit of Racah coefficients", in Spectroscopy and group theoretical methods in Physics, F. Bloch ed. (North-Holland, Amsterdam, 1968).

[6] J. C. Baez, J. D. Christensen and G. Egan, "Asymptotics of 10j symbols," Class. Quant. Grav. 19, 6489 (2002) [arXiv:gr-qc/0208010].

[7] J. W. Barrett and C. M. Steele, "Asymptotics of relativistic spin networks," Class. Quant. Grav. 20, 1341 (2003) [arXiv:gr-qc/0209023].

L. Freidel and D. Louapre, "Asymptotics of 6j and 10j symbols," Class. Quant. Grav. 20 (2003) 1267 [arXiv:hep-th/0209134].

[8] J. Hackett and S. Speziale, "Grasping rules and semiclassical limit of the geometry in the Ponzano-Regge model," Class. Quant. Grav. 24 (2007) 1525 [arXiv:gr-qc/0611097].

[9] L. Freidel and D. Louapre, "Ponzano-Regge model revisited. I: Gauge fixing, observables and interacting spinning particles," Class. Quant. Grav. 21 (2004) 5685 [arXiv:hep-th/0401076].

[10] L. Freidel and E. R. Livine, "Effective 3d quantum gravity and non-commutative quantum field theory," Phys. Rev. Lett. 96, 221301 (2006) [arXiv:hep-th/0512113].

M. Karadi, E. R. Livine, D. Oriti and J. Ryan, "Effective non-commutative field theory for spinning particles coupled to $3 \mathrm{~d}$ quantum gravity," to appear. 
[11] T. Thiemann, "QSD V: Quantum gravity as the natural regulator of matter quantum field theories," Class. Quant. Grav. 15, 1281 (1998) [arXiv:gr-qc/9705019].

K. Noui and A. Perez, "Three dimensional loop quantum gravity: Coupling to point particles," Class. Quant. Grav. 22 (2005) 4489 [arXiv:gr-qc/0402111].

[12] L. Freidel, D. Oriti and J. Ryan, "A group field theory for 3d quantum gravity coupled to a scalar field," arXiv:gr-qc/0506067.

W. J. Fairbairn and E. R. Livine, "3d spinfoam quantum gravity: Matter as a phase of the group field theory," arXiv:gr-qc/0702125.

[13] D. Oriti and H. Pfeiffer, "A spin foam model for pure gauge theory coupled to quantum gravity," Phys. Rev. D 66, 124010 (2002) [arXiv:gr-qc/0207041].

A. Mikovic, "Spin foam models of Yang-Mills theory coupled to gravity," Class. Quant. Grav. 20, 239 (2003) [arXiv:gr-qc/0210051].

[14] L. Freidel and K. Krasnov, "Spin foam models and the classical action principle," Adv. Theor. Math. Phys. 2 (1999) 1183 [arXiv:hep-th/9807092].

[15] W. J. Fairbairn, "Fermions in three-dimensional spinfoam quantum gravity," arXiv:gr-qc/0609040.

[16] D. Oriti, C. Rovelli and S. Speziale, "Spinfoam 2d quantum gravity and discrete bundles," Class. Quant. Grav. 22 (2005) 85 [arXiv:gr-qc/0406063].

[17] S. Deser, R. Jackiw and G. 't Hooft, "Three-Dimensional Einstein Gravity: Dynamics Of Flat Space," Annals Phys. 152 (1984) 220.

[18] E. Witten, "(2+1)-Dimensional Gravity As An Exactly Soluble System," Nucl. Phys. B 311 (1988) 46.

[19] M. B. Halpern, "Field Strength Formulation Of Quantum Chromodynamics," Phys. Rev. D 16 (1977) 1798.

M. Schaden, H. Reinhardt, P. A. Amundsen and M. J. Lavelle, "An Effective Action For Yang-Mills Field Strengths," Nucl. Phys. B 339 (1990) 595.

[20] A. Accardi, A. Belli, M. Martellini and M. Zeni, "Cohomology and renormalization of BFYM theory in three dimensions," Nucl. Phys. B 505 (1997) 540 [arXiv:hep-th/9703152].

A. S. Cattaneo, P. Cotta-Ramusino, F. Fucito, M. Martellini, M. Rinaldi, A. Tanzini and M. Zeni, "Fourdimensional Yang-Mills theory as a deformation of topological BF theory," Commun. Math. Phys. 197 (1998) 571 [arXiv:hep-th/9705123].

[21] C. Rovelli and S. Speziale, "On the perturbative expansion of a quantum field theory around a topological sector," Gen. Rel. Grav. 39 (2007) 167 [arXiv:gr-qc/0508106].

[22] F. Conrady, "Analytic derivation of gluons and monopoles from SU(2) lattice Yang-Mills theory. I: BF Yang-Mills representation," arXiv:hep-th/0610236.

[23] R. De Pietri, L. Freidel, K. Krasnov and C. Rovelli, "Barrett-Crane model from a Boulatov-Ooguri field theory over a homogeneous space," Nucl. Phys. B 574 (2000) 785 [arXiv:hep-th/9907154].

L. Freidel, "Group field theory: An overview," Int. J. Theor. Phys. 44 (2005) 1769 [arXiv:hepth/0505016].

D. Oriti, "The group field theory approach to quantum gravity," arXiv:gr-qc/0607032.

[24] M. P. Reisenberger, "A lattice worldsheet sum for 4-d Euclidean general relativity," arXiv:grqc/9711052.

[25] R. De Pietri and C. Rovelli, "Geometry Eigenvalues and Scalar Product from Recoupling Theory in Loop Quantum Gravity," Phys. Rev. D 54 (1996) 2664 [arXiv:gr-qc/9602023].

[26] V. G. Turaev and O. Y. Viro, "State sum invariants of 3 manifolds and quantum 6j symbols," Topology 31 (1992) 865. 
[27] C. Rovelli and S. Speziale, "Reconcile Planck-scale discreteness and the Lorentz-Fitzgerald contraction," Phys. Rev. D 67 (2003) 064019 [arXiv:gr-qc/0205108].

E. R. Livine and D. Oriti, "About Lorentz invariance in a discrete quantum setting," JHEP 0406 (2004) 050 [arXiv:gr-qc/0405085].

[28] J. Collins, A. Perez, D. Sudarsky, L. Urrutia and H. Vucetich, "Lorentz invariance: An additional finetuning problem," Phys. Rev. Lett. 93 (2004) 191301 [arXiv:gr-qc/0403053].

G. Amelino-Camelia, "Introduction to quantum-gravity phenomenology," Lect. Notes Phys. 669 (2005) 59 [arXiv:gr-qc/0412136].

F. Girelli, E. R. Livine and D. Oriti, "Deformed special relativity as an effective flat limit of quantum gravity," Nucl. Phys. B 708 (2005) 411 [arXiv:gr-qc/0406100].

T. Jacobson, S. Liberati and D. Mattingly, "Lorentz violation at high energy: Concepts, phenomena and astrophysical constraints," Annals Phys. 321 (2006) 150 [arXiv:astro-ph/0505267].

[29] G. C. Rossi and M. Testa, "The Structure Of Yang-Mills Theories In The Temporal Gauge. 1. General Formulation," Nucl. Phys. B 163 (1980) 109.

[30] F. Mattei, C. Rovelli, S. Speziale and M. Testa, "From 3-geometry transition amplitudes to graviton states," Nucl. Phys. B 739, 234 (2006) [arXiv:gr-qc/0508007].

[31] B. Dittrich, L. Freidel and S. Speziale, "Linearized dynamics from the 4-simplex Regge action," arXiv:0707.4513 [gr-qc]. 